\section{Ketorolac vs. morfina nel trattamento del dolore acuto in pazienti traumatizzati: un'analisi di minimizzazione dei costi}

Colombo G.L.II, Borghi B.\#, Caruggi M.¥, De Nicola A.ф

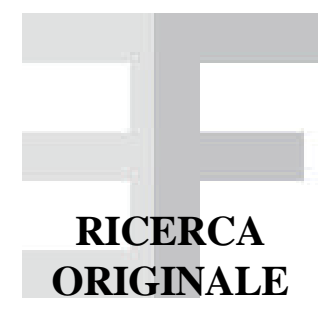

\begin{abstract}
Aim of this study is to develop an economic valuation comparing ketorolac vs. morphine for the treatment of sharp pain caused by heavy limb injury, in traumatology ward and in first aid ward.

As cost minimization analysis, this economic valuation has been based on clinical results of Rainer et al. (2000). Ketorolac and morphine have the same efficacy in calming the pain; the greatest difference in the clinical outcomes is about the morphine side effects (nausea, vomiting and phlebitis).

Assuming the hospital point of view with a differential costs perspective, the ketorolac patients showed two consequences than could generate lower resources consumption: less side effects and the reduction of time permanence in first aid ward.

Considering the drug cost, the administration cost and the side effects treatment costs, the expenditure for every single morphine patient is L. 23.385 , vs L. 4.875 for a ketorolac patient, with a difference of L. 18.510 .
\end{abstract}

Farmeconomia e percorsi terapeutici 2001; 2 (2): $91-98$

\section{INTRODUZIONE}

I pazienti che normalmente si presentano al Pronto Soccorso o al reparto di traumatologia con dolore acuto a seguito di una lesione ad un arto, necessitano di un trattamento con analgesici efficaci (Yealy et al, 1992., Goodacre et al, 1997). L'uso di questi farmaci nei reparti di Pronto Soccorso e nelle unità di terapia intensiva non appare sempre ottimale. Alcuni analgesici come la morfina, possono causare dipendenza e, in dosi singole, determinare gravi effetti collaterali che richiedono monitoraggio e cure ulteriori da parte di medici ed infermieri. Perciò questi farmaci anche se relativamente poco costosi, possono incidere pesantemente sulle risorse del Sistema Sanitario (British Medical Association 1997; Benedetti C. et al, 1990). I FANS sono altrettanto efficaci nell'alleviare il dolore sia moderato che acuto e hanno dimostrato di avere meno effetti collaterali che gli oppiacei (Gillis et al, 1997, Brown et al, 1990). Nel Nord America, in Italia e in altri Paesi Europei, il ketorolac (ketorolac trometamina) è l'unico FANS autorizzato ed attualmente utilizzato per la cura del dolore mediante somministrazione endovenosa.
Attualmente la terapia analgesica con morfina, somministrata per via endovenosa, risulta ancora la più impiegata, rispetto all'uso di altri potenti analgesici (Cordal et al, 1996; British Medical Association, 1997); tuttavia l'effettivo impatto economico relativo al suo utilizzo è poco conosciuto.

Obiettivo del presente lavoro è quello di sviluppare una valutazione economica confrontando il ketorolac vs. morfina nel trattamento del dolore acuto causato da lesioni gravi ad un arto, in un reparto di traumatologia e in un Pronto Soccorso. Tramite l'identificazione di tutte le principali voci di costo associate ad una terapia farmacologica, viene identificato il costo effettivo di trattamento di questi pazienti. L'analisi economica è stata condotta, come analisi di minimizzazione dei costi, includendo i risultati di un lavoro clinico di Rainer et al. 2000. Questo studio è stato condotto secondo le recenti linee guida definite per i revisori del British Medical Journal (BMJ), al fine di rispecchiare, per quanto possibile, una realtà internazionale. L'efficacia del ketorolac e della morfina è stata valutata in termini di riduzione della sintomatologia dolorifica. Il punto di vista adottato nell'analisi dei costi
II S.A.V.E. Studi Analisi Valutazioni Economiche, Milano \# Ospedale Rizzoli,Bologna $¥$ Unità di Terapia del Dolore, Ospedale S. Leonardo,

Castellammare di Stabia, Napoli ф Azienda Ospedaliera "Ospedale Civile di Legnano”, Legnano 
è quello dell'ospedale; questo al fine di valutare quale delle due alternative sia in grado di determinare una migliore gestione dei pazienti a costi più contenuti.

\section{MATERIALI E METODI}

Per questa analisi ci si è riferiti allo studio di Rainer et al. 2000, in cui è stata valutata l'efficacia clinica ed il consumo di risorse legate all'uso di morfina e ketorolac. Sono stati arruolati 182 pazienti con età maggiore di 16 anni, che si sono presentati, in sequenza temporale e nell' arco di una settimana, al Pronto Soccorso presentando lesione dolorosa acuta ad un arto. Sono stati esclusi i pazienti che avevano avuto precedenti di abuso di stupefacenti, demenza, indigestione, ulcera peptica o emorragia gastrointestinale, problemi di coagulazione, gravidanze o effetti collaterali al ketorolac o alla morfina, insufficienza renale o cardiaca, problemi epatici, sanguinamenti rettali, uso recente $(<24$ ore $)$ di FANS, asma, patologie croni- che di ostruzione delle vie aeree, sindromi dolorifiche croniche, o trattamenti precedenti con analgesici per lo stesso evento. Tali pazienti sono stati quindi randomizzati in uno dei due gruppi di trattamento. Le caratteristiche dei pazienti arruolati, le cause della lesione e il tipo di frattura e/o lesione sono sintetizzati in Tabella 1.

Alla prima coorte è stato somministrato ketorolac alla concentrazione di $2 \mathrm{mg} / \mathrm{ml}$ di soluzione, mentre alla seconda coorte è stata somministrata morfina ad una concentrazione di $1 \mathrm{mg} / \mathrm{ml}$ di soluzione. Ciascun paziente del primo gruppo ha ricevuto $10 \mathrm{mg} \mathrm{(5}$ $\mathrm{ml}$ ) di ketorolac per endovenosa somministrato in un tempo superiore ai 20 secondi, seguito da una dose di $5 \mathrm{mg}(2,5 \mathrm{ml})$ ogni 5 minuti fino a 20 minuti (per un massimo di 30 $\mathrm{mg}$ ) secondo la necessità. I pazienti del secondo gruppo hanno ricevuto morfina per endovenosa una dose di $5 \mathrm{mg}(5 \mathrm{ml})$ somministrata in un tempo superiore ai 60 secondi seguita da una dose di $2.5 \mathrm{mg}(2,5 \mathrm{ml})$ ogni 5 minuti fino 20 minuti (con un massimo di 15 $\mathrm{mg})$ secondo la necessità.

\begin{tabular}{|c|c|c|c|}
\hline VARIABILI & $\begin{array}{c}\text { KETOROLAC } \\
(n=75)\end{array}$ & $\begin{array}{c}\text { MORFINA } \\
(n=73)\end{array}$ & $\mathbf{p}$ \\
\hline Età media (anni) e (SD) & $53,9(21,7)$ & $53,2(21,8)$ & 0,86 \\
\hline $\mathrm{N}^{\circ}(\%)$ di uomini & $36(51)$ & $33(45)$ & 0,516 \\
\hline Media (SD) massa del corpo indicata come $\mathrm{Kg} / \mathrm{m} 3$ & $222,8(3,2)$ & $23,0(3,7)$ & 0,77 \\
\hline Tempo medio tra la lesione e l'arrivo in ospedale & $95(30-690)$ & $82(33-921)$ & 0,75 \\
\hline \multicolumn{4}{|l|}{ Causa della lesione: } \\
\hline Incidente con veicolo a motore & $6(6)$ & $4(5)$ & 0,58 \\
\hline Cadute & $46(61)$ & $51(70)$ & \\
\hline Urti & $20(27)$ & $14(19)$ & \\
\hline Altro & $3(4)$ & $4(5)$ & \\
\hline Fratture: & $50(67)$ & $46(66)$ & 0,91 \\
\hline Clavicola, Omero, & $5(7)$ & $8(11)$ & \\
\hline Radio, Ulna & $8(11)$ & $11(15)$ & \\
\hline Mano & $15(20)$ & $13(16)$ & \\
\hline Femore & $14(19)$ & $12(16)$ & \\
\hline Tibia, Perone & $5(7)$ & $3(4)$ & \\
\hline Piede & $2(3)$ & $1(1)$ & \\
\hline \multicolumn{4}{|l|}{ Lesioni senza fratture } \\
\hline Slogamento arto inferiore & $2(3)$ & $1(1)$ & \\
\hline Lesione all'articolazione dell'arto inferiore & $10(13)$ & $10(14)$ & \\
\hline Lesione all'articolazione dell'arto superiore & $14(19)$ & $14(19)$ & \\
\hline \multicolumn{4}{|l|}{ Valore iniziale medio del dolore: } \\
\hline A riposo & $3,8(1,1)$ & $3,9(1,1)$ & 0,65 \\
\hline In movimento & $8,1(1,2)$ & $8,1(1,2)$ & 0,85 \\
\hline Riferito ad accertamento ortopedico & $41(55)$ & $36(49)$ & $\overline{0,52}$ \\
\hline Ricovero successivo in ospedale & $38(51)$ & $29(40)$ & 0,16 \\
\hline Ricovero con effetti collaterali & 0 & $3(4)$ & \\
\hline
\end{tabular}

Tabella 1

Caratteristiche dei pazienti arruolati Fonte: Rainer et al. 2000 . 
I principali outcomes clinici sono stati la misurazione del sollievo dal dolore, calcolata come variazione del VAS (Visual Analogue Scale) e gli effetti collaterali dei due analgesici. Per determinare il punteggio base del dolore e negli intervalli di tempo successivi alla prima iniezione, si è utilizzata una scala analogica visuale di 10 pollici $(254 \mathrm{~mm})$, numerata da 0-10. Le osservazioni di routine, il punteggio del dolore e gli effetti collaterali, sono stati registrati ogni 5 minuti per i primi 30 minuti dopo la somministrazione dell'analgesia e ogni 30 minuti per un ora e mezza successiva, ed un'altra volta dopo sei ore. Il sollievo dal dolore è stato valutato sia al raggiungimento del $50 \%$, del $75 \%$ e del $100 \%$ di riduzione del punteggio del dolore (a riposo e in attività) sia come media delle variazioni del punteggio.

\section{CONSUMO DI RISORSE}

La realizzazione di una terapia farmacologica implica non solo il costo del consumo del prodotto, ma anche di altre risorse economiche che possono rappresentare, talvolta, un costo non trascurabile per l'ospedale (Eisemberg et al, 1988; Eandi et al, 1993). I fattori di costo diretto associati ad un trattamento farmacologico sono: costo di acquisto del prodotto farmaceutico, costo di stoccaggio, costo del materiale per la preparazione e somministrazione del farmaco, costo dello smaltimento rifiuti, costo del lavoro di medici ed infermieri per la preparazione e somministrazione dei farmaci, costo del monitoraggio generale e terapeutico, costo delle reazioni avverse e dell'insuccesso terapeutico (Drummond et al, 1997; Jefferson et al, 1998).

Per tale lavoro si sono adottate le stime di consumo di risorse sanitarie presenti nello studio di Rainer et al 2000, applicandole alla realtà ospedaliera italiana. Il confronto tra i due trattamenti è stato effettuato a costi differenziali. La metodologia a costi differenziali permette infatti di concentrarsi solo su quelle risorse (e, quindi, su quei costi) che presentano, o che potenzialmente possono presentare, variazioni tra le diverse alternative in esame, evitando al ricercatore l'onere di misurare l'ammontare di quegli elementi che si presentano uguali in entrambe le alternative. I costi sono stati calcolati in base alle operazioni richieste per la preparazione e somministrazione degli analgesici e di altri farmaci e la cura degli effetti collaterali. Il punto di vista adottato per la valutazione dei costi è quello dell'ospedale. Non si sono considerati, perciò, i costi avvertibili solo da altri soggetti, quali ad esempio il paziente e/ o la società nel suo complesso.

La morfina cloridrato è venduta al pubblico in diverse concentrazioni e confezioni. Alla concentrazione utilizzata per lo studio ( $1 \mathrm{mg} / 1 \mathrm{ml}$ di soluzione), il prezzo al pubblico di una fiala è pari a L.3.100. Per ciascun paziente sono necessarie una fiala e mezzo per un totale di L.4.650. Essendo il farmaco utilizzato in ospedale, è soggetto ad uno sconto del $50 \%$; il prezzo per paziente risulta così pari a L.2.325. Il ketorolac (Tora-dol, Recordati) è venduto al pubblico in confe-

\begin{tabular}{|lcccc}
\hline & $\begin{array}{c}\text { Ketorolac } \\
(\mathbf{n = 7 5})\end{array}$ & $\begin{array}{c}\text { Tempo per la } \\
\text { somministrazione }\end{array}$ & $\begin{array}{c}\text { Morfina } \\
(\mathbf{n = 7 3})\end{array}$ & $\begin{array}{c}\text { Tempo per la } \\
\text { somministrazione }\end{array}$ \\
\hline \hline Dose d'attacco & $\begin{array}{c}10 \mathrm{mg} \\
(5 \mathrm{ml})\end{array}$ & $20 \mathrm{sec}$. & $\begin{array}{c}5 \mathrm{mg} \\
(5 \mathrm{ml})\end{array}$ & $60 \mathrm{sec}$. \\
\hline Dosi successive & $\begin{array}{c}5 \mathrm{mg} \\
(2,5 \mathrm{ml})\end{array}$ & $\begin{array}{c}\text { ogni } 5 \mathrm{~min} . \\
\text { Max } 20 \mathrm{~min} .\end{array}$ & $\begin{array}{r}2,5 \mathrm{mg} \\
(2,5 \mathrm{ml})\end{array}$ & $\begin{array}{r}\text { ogni } 5 \mathrm{~min} . \\
\text { Max 20 min. }\end{array}$ \\
\hline \hline Dose Max & $30 \mathrm{mg}$ & & $15 \mathrm{mg}$ & \\
\hline $\begin{array}{l}\text { Costo d'acquisto } \\
\text { dell'analgesico } \\
\text { (per confezione) }\end{array}$ & \begin{tabular}{c} 
(a) \\
\hline Cos diale da $30 \mathrm{mg})$
\end{tabular} & & $\begin{array}{c}3.100 \\
\text { per fiala }\end{array}$ & \\
\hline
\end{tabular}

\begin{tabular}{lll}
\hline $\begin{array}{l}\text { Costo dell'analgesico } \\
\text { per paziente }\end{array}$ & 1.733 & 2.325 \\
\hline
\end{tabular}

\begin{tabular}{|lllll}
\hline $\begin{array}{l}\text { Costo per la preparazione } \\
\text { somministrazione del farmaco } \\
\text { (costo del personale) }\end{array}$ & 3.300 & $5 \mathrm{~min}$. & 6.600 & $10 \mathrm{~min}$. \\
\hline
\end{tabular}

\begin{tabular}{|c|c|c|}
\hline $\begin{array}{l}\text { Costo medio } \\
\text { per paziente }\end{array}$ & 5.033 & 8.925 \\
\hline
\end{tabular}

Tabella 2

Costi e dosaggi della terapia farmacologica (a) Prezzo al pubblico. Fonte: elaborazioni Costo totale pazienti arruolati $\quad 377.500$ 651.525 da Rainer et al, 2000. 
zione da 3 fiale da $30 \mathrm{mg}$. Per ciascun paziente è necessaria una confezione il cui prezzo al pubblico è di L.10.400. Il costo del Ketorolac per uso ospedaliero è di L.1.733 a fiala, che corrisponde anche al prezzo medio per paziente.

Il costo orario del lavoro di medici ed infermieri è stato utilizzato sia per il calcolo del costo della somministrazione, sia per determinare il costo per il trattamento degli effetti collaterali dei pazienti. Il costo del personale medico è stato calcolato sulla base del costo medio annuo per l'ospedale (L.160.000.000), suddiviso per 42 settimane lavorative e per le 34 ore di lavoro a settimana (38 contrattuali al netto di quattro ore di aggiornamento obbligatorio). Il costo del lavoro dell'infermiere è stato determinato suddividendo lo stipendio annuo (L.60 milioni) per le 42 settimane e per le 36 ore di lavoro. La somministrazione di morfina richiede un tempo maggiore valutato in cinque minuti. La differenza di tempo si traduce così in un costo aggiuntivo pari a L.3.300 per ciascun paziente che, per i 73 pazienti che hanno assunto morfina equivale a L.240.900.

\section{RISULTATI}

L'efficacia dei due farmaci nel sedare il dolore è risultata sovrapponibile nei due gruppi di confronto (con una maggiore efficacia della morfina nel ridurre il dolore con l'arto fermo rispetto ad una migliore efficacia di ketorolac nel ridurre il dolore con l'arto in movimento). La maggiore differenza riscontrabile negli outcomes clinici (e di conseguenza economici) riguarda invece la frequenza di comparsa degli effetti collaterali. Gli effetti collaterali sono risultati ben 16 volte superiori nel gruppo di pazienti che hanno assunto morfina vs. ketorolac, con una differenza di 144,2 (95\% intervallo di confidenza da 41,5 a 501,6; $\mathrm{p}<0,0001)$.

Come sintetizzato in Tab. 3 , i più frequenti effetti collaterali sono risultati: sonnolen- za, sonno, vertigini, nausea e vomito. Sonno e sonnolenza hanno riguardato 51 pazienti (il $70 \%$ ) a cui è stata somministrata morfina vs. un paziente (1\%) del gruppo ketorolac. La nausea ha riguardato 55 pazienti $(75 \%)$ in morfina vs. i due pazienti ( $2 \%$ circa) in ketorolac. Sonnolenza e sonno non comportano, in genere, un consumo aggiuntivo di risorse; in alcuni casi si possono considerare addirittura fenomeni positivi in quanto rendono più gestibile il paziente. Anche le vertigini non comportano un consumo aggiuntivo di risorse dal momento che il paziente è già normalmente sdraiato sul lettino.

Nausea, vomito e flebite si sono rivelati gli effetti collaterali più gravi nella coorte che ha assunto morfina. Tali effetti collaterali sono gestiti in genere con la somministrazione di antiemetici per la nausea e vomito, mentre per la flebite viene generalmente indicato un FANS e una pomata antidolorifica. Come antiemetico si è ipotizzato di utilizzare due fiale di metocopramide $10 \mathrm{mg}$ per paziente che, al prezzo di acquisto ospedaliero corrisponde a un costo di L.1.020 per paziente con evento. In termini di personale sanitario, si possono stimare i costi per il personale infermieristico, che ha osservato e comunicato al medico l'episodio collaterale. Il tempo stimato va da due a cinque minuti per effetto collaterale, con una media di tre minuti per paziente interessato dall'effetto collaterale; per la somministrazione del farmaco (con infusione endovenosa già in corso) dai cinque ai 10 minuti, in media otto minuti per paziente. In totale sono necessari 11 minuti che, al costo medio di L.660 al minuto, equivalgono a circa L.7.200 per paziente con evento. Il tempo del personale medico per la valutazione e prescrizione varia tra un minimo di cinque minuti e un massimo di 10 , con un tempo medio di otto minuti per paziente interessato dall'effetto. Considerando un costo medio di L.1.870 al minuto, ciascun paziente con evento costa circa

\section{Tabella 3}

Numero e percentuale di pazienti con effetti collaterali Fonte: Rainer et al. 2000 .

\begin{tabular}{|c|c|c|c|c|c|}
\hline & \multicolumn{2}{|c|}{ KETOROLAC $(n=75)$} & \multicolumn{2}{|c|}{ MORFINA $(n=73)$} & \multirow[b]{2}{*}{$\mathbf{p}$} \\
\hline & $\mathbf{N}^{\circ}$ eventi & $\%$ & $\mathbf{N}^{\circ}$ eventi & $\%$ & \\
\hline Sonnolenza & 1 & 1 & 43 & 59 & $<0,0001$ \\
\hline Sonno & 0 & & 8 & 11 & $<0,0054$ \\
\hline Vertigini & 2 & 2 & 55 & 75 & $<0,0001$ \\
\hline Nausea & 0 & 0 & 27 & 37 & $<0,0001$ \\
\hline Vomito & 0 & 0 & 12 & 16 & $<0,0010$ \\
\hline Flebite & 0 & 0 & 20 & 27 & $<0,0001$ \\
\hline Totale & 4 & 6 & 65 & 69 & $<0,0001$ \\
\hline
\end{tabular}




\begin{tabular}{|c|c|c|c|c|}
\hline VARIABILI & $\begin{array}{c}\text { KETOROLAC } \\
(n=75)\end{array}$ & $\begin{array}{c}\text { MORFINA } \\
\quad(n=73)\end{array}$ & $\begin{array}{c}\text { DIFFERENZA } \\
\text { (intervallo di } \\
\text { confidenza del } \\
95 \% \text { ) }\end{array}$ & $\mathbf{p}$ \\
\hline \multirow[t]{2}{*}{$\begin{array}{l}\text { Intervallo tra } \\
\text { l'arrivo al Pronto } \\
\text { Soccorso e la } \\
\text { prescrizione } \\
\text { medica } \\
\text { dell'analgesico } \\
\end{array}$} & 38,0 & 39,0 & 1,0 & 0,72 \\
\hline & $($ da 30,0 a 54,0$)$ & (da 29,0 a 53 ) & $($ da 5,0 a 7,0$)$ & \\
\hline \multirow[t]{2}{*}{$\begin{array}{l}\text { Tempo di } \\
\text { preparazione della } \\
\text { terapia analgesica }\end{array}$} & 5,0 & 10,0 & 2,0 & 0,0002 \\
\hline & (da 5,0 a 10,0$)$ & (da 5,5 a 12,5$)$ & $(\mathrm{da} 0,5)$ & \\
\hline \multirow[t]{2}{*}{$\begin{array}{l}\text { Esame } \\
\text { radiografico }\end{array}$} & 5,0 & 5,0 & 0,0 & 0,75 \\
\hline & $($ da 5,0 a 10,0$)$ & $(\mathrm{da} 4,0$ a 10,0$)$ & (da 1,0 a 0$)$ & \\
\hline \multirow[t]{2}{*}{$\begin{array}{l}\text { Tempo totale } \\
\text { trascorso in } \\
\text { Pronto Soccorso }\end{array}$} & 155,0 & 171,0 & 15,0 & 0,11 \\
\hline & (da 112 a 198,0$)$ & (da 126 a 208,5$)$ & (da 4,0 a 33) & \\
\hline
\end{tabular}

\begin{tabular}{llcc}
\hline $\begin{array}{l}\text { Intervallo di tempo } \\
\text { tra la } \\
\text { somministrazione }\end{array}$ & 130,0 & 20,0 & 0,02 \\
$\begin{array}{l}\text { dell'analgesico e la } \\
\text { dimissione dal }\end{array}$ & & & \\
Pronto Soccorso & 115,0 & & \\
\hline
\end{tabular}

$\begin{array}{llll}(\text { da } 75,0 \text { a } 149,0) \quad(\text { da } 95 \text { a } 170) \quad(\text { da } 4,0 \text { a } 39,0) & 2000 .\end{array}$

L.15.000. Il costo per paziente con nausea e vomito, comprensivo del costo del farmaco, è risultato pari a L.23.200 per paziente con evento. I sintomi nausea e vomito, si sono presentati in 39 pazienti sui 73 (il 53\%) del gruppo trattato con morfina, per complessive L. 908.580, e nessuno nel gruppo ketorolac.

La flebite, intesa come forma blanda della patologia, facilmente risolvibile, viene generalmente trattata con la somministrazione di una pomata antiflebitica (Lasoven gel), con un costo ospedaliero di circa L. 900. L'applicazione locale della pomata, effettuato da un'infermiera, è stato calcolato in un tempo che può variare tra i cinque e i 15 minuti, con una media di 10; questo determina un costo complessivo per paziente con flebite pari a L.7.500; ciò comporta un costo complessivo per tale evento pari a L.150.000 per i complessivi 20 pazienti con flebite (il 27\%) del gruppo trattato con morfina. Si può quindi stimare il risparmio potenziale di risorse con ketorolac per evento evitato pari a L.2.054 per paziente arruolato. Si sospende infine, la valutazione sulla maggior incidenza dei ricoveri in ospedale poiché è da verificare in quale misura tale variabile dipenda dalla gravità intrinseca del caso, piuttosto che dalla somministrazione dell' analgesico. I pazienti nel gruppo morfina hanno atteso infine per un periodo temporale maggiore tra il momento della prescrizione e la somministrazione della dose iniziale vs. il gruppo ketorolac $(\mathrm{p}=0,0002)$ e di conseguenza rimangono più a lungo in Pronto Soccorso (con una differenza non statisticamente significativa $\mathrm{P}=0,11)$.

Il ketorolac ha dimostrato di ridurre il tempo trascorso tra la somministrazione dell'anal-gesico e la dimissione dal Pronto Soccorso. Tra la somministrazione dell' analgesico e la dimissione dal Pronto Soccorso si osserva una differenza tra i valori mediani pari a 15-20 minuti a favore dei pazienti trattati con ketorolac. Tale fatto non può tradursi immediatamente in minori costi monetari per la struttura ospedaliera, quanto piuttosto in una migliore accoglienza dei pazienti che affrontano un ambiente meno congestionato e che possono essere osservati in tempi più brevi. Ne deriva un sicuro guadagno in immagine per il servizio di Pronto Soccorso, purtroppo ad oggi non monetizzabile, ma
Tabella 4

Numero di minuti per il trattamento dei pazienti (mediana) Fonte: elaborazioni da Rainer et al. 2000. 
Tabella 5

Risultati dell'analisi.

\begin{tabular}{|lrrrrr}
\hline & $\begin{array}{c}\text { Ketorolac } \\
(\mathbf{n = 7 5})\end{array}$ & $\boldsymbol{\%}$ & $\begin{array}{c}\text { Morfina } \\
(\mathbf{n = 7 3 )}\end{array}$ & \% & Differenza \\
\hline \hline Costo della terapia farmacologica & 130.000 & $32 \%$ & 169.725 & $10 \%$ & -39.725 \\
\hline Costo della somministrazione & 247.500 & $68 \%$ & 481.800 & $28 \%$ & -234.300 \\
\hline Costo degli effetti collaterali & - & $0 \%$ & 1.055 .580 & $62 \%$ & -1.055 .580 \\
\hline Nausea, vomito & - & $0 \%$ & 905.580 & $53 \%$ & -905.580 \\
\hline Flebite & - & $0 \%$ & 150.000 & $9 \%$ & -150.000 \\
\hline Costo totale dei pazienti arruolati & 377.500 & $100 \%$ & 1.707 .105 & $100 \%$ & -1.329 .605 \\
\hline Costo medio per paziente arrualato & 5.033 & & 23.385 & & -18.352
\end{tabular}

forse più misurabile con idonei strumenti di customer satisfaction.

Escludendo i costi per accesso al Pronto Soccorso, la differenza tra i due gruppi è dovuta al costo d'acquisto del farmaco e alla cura degli effetti collaterali. Il costo del ketorolac è risultato complessivamente di $\mathrm{L}$. 130.000 (L.1.733 per paziente) vs. L. 169.725 della morfina (L. 2.325 per paziente). Al costo del farmaco, occorre sommare il costo per la preparazione e somministrazione L. 247.500 e L. 481.800 rispettivamente (pari a L.3.300 e L.6.600 per paziente arruolato). La morfina ha determinato un rilevante numero di effetti collaterali che hanno richiesto un maggiore consumo di risorse sanitarie (farmaci e/o personale medico). Il costo per paziente con effetti collaterali è risultato complessivamente di L. 1.055.580 (L.14.460 per paziente). Considerando il costo del farmaco, il costo per la somministrazione ed il costo per il trattamento degli effetti collaterali un paziente in morfina genera un costo di L. 23.385, vs. L.5.033 di un paziente ketorolac, con una differenza di L.18.352 per paziente. La differenza complessiva in termini assoluti tra le due coorti risulta pari a L.1.329.605 (Tab. 5).

\section{DISCUSSIONE}

La metodologia di valutazione economica adottata nel presente lavoro è la minimizzazione dei costi. Tale analisi si basa sul presupposto che il grado di efficacia delle due modalità alternative farmacologiche sia identico o, perlomeno, non significativamente diverso, e si procede quindi confrontando i costi connessi alle due alternative dal punto di vista dell'ospedale (Freund et al., 1992; Sloan F. A., 1995).

I risultati clinici dello studio di Rainer et al. 2000, all'interno dei quali la presente valutazione economica è stata sviluppata, hanno infatti evidenziato una sostanziale sovrapposizione dell' efficacia clinica dei due trattamenti. L'efficacia dei due farmaci nel sedare il dolore è risultata sovrapponibile nei due gruppi, mentre la maggiore differenza riscontrabile negli outcomes clinici (e di conseguenza economici) riguarda invece la frequenza di comparsa degli effetti collaterali. Il trattamento di tali eventi avversi può tradursi infatti in perdite o guadagni di risorse a seconda del punto di vista da cui si osservano e a seconda di alcune ipotesi organizzative. Assumendo il punto di vista dell'ospedale, adottando una prospettiva a costi differenziali (che significa considerare la variazione dei costi per il trattamento o non trattamento di un paziente incrementale) e ipotizzando l'attuale piena occupazione della capacità produttiva del personale, si può ritenere che nel gruppo dei pazienti trattati con ketorolac si siano evidenziate, tra le altre, due conseguenze che possono tradursi in un minore consumo di risorse rispetto al gruppo di pazienti trattati con morfina. La prima conseguenza è il minor numero di pazienti con effetti collaterali; l'altra conseguenza è la riduzione del tempo di preparazione dell' analgesia e la riduzione del tempo trascorso tra somministrazione dell'analgesico e dimissione dal Pronto Soccorso e, di riflesso, riduzione del tempo totale trascorso al Pronto Soccorso.

Escludendo i costi per accesso al pronto soccorso, la differenza maggiore tra i due gruppi è dovuta al costo d'acquisto del farmaco e al trattamento degli effetti collaterali. Il costo del ketorolac è di L.1.733 per paziente vs. L. 2.325 della morfina. Al costo del farmaco, occorre aggiungere il costo per la somministrazione; la somministrazione del ketorolac costa L.3.300 vs. L.6.600 della morfina. Il costo totale dell' analgesia è di L.5.033 per un paziente in ketorolac e L.8.925 per un paziente in morfina, con una differenza di L.3.892 a favore del ketorolac. La morfina ha provocato, inoltre, un numero rilevante di effetti collaterali che hanno richiesto l'utilizzo di farmaci e di personale medico. Il costo per il trattamento degli effetti collaterali della morfina è risultato pari a L. 1.055.580 (L.14.460 per paziente), mentre pari a zero sono risultati i costi per il trattamento degli 
effetti collaterali con ketorolac. Gli effetti collaterali presenti nel gruppo del ketorolac sono stati soltanto sonnolenza, sonno e vertigini; tali effetti collaterali non richiedono, in genere, nessuna terapia farmacologica e nessun costo per l'assistenza medica e infermieristica.

L'obiettivo di questo lavoro era quello di confrontare costi e conseguenze dell'impiego di ketorolac vs. morfina, al fine di evidenziare eventuali vantaggi clinici ed economici della scelta nel contesto sanitario italiano. Dal punto di vista strettamente operativo tutto ciò determina, come abbiamo appena analizzato, una riduzione dei costi grazie al risparmio di tempo per la preparazione e la somministrazione e trattamento degli effetti collaterali. In questo caso la maggiore praticità nella modalità di approntamento $\mathrm{e}$ somministrazione del prodotto favorisce una migliore organizzazione del contesto lavorativo dell'ospedale, offrendo nel contempo anche una migliore qualità alla prestazione erogata.

Il trattamento del dolore rimane infatti uno degli obiettivi principali dei reparti di Pronto Soccorso di tutto il mondo; in questa prospettiva le linee di condotta nella terapia analgesica basate sulla rapidità di azione, sicurezza e costo-efficacia sono essenziali per la buona salute e benessere dei pazienti. Il ketorolac ha dimostrato di provocare minori effetti collaterali rispetto alla morfina, di ridurre il tempo di permanenza nel reparto e di richiedere minore tempo di assistenza da parte di medici ed infermieri. Risulta particolarmente importante la riduzione del tempo trascorso dai pazienti che assumono ketorolac nel Pronto Soccorso; la maggiore domanda e i prolungati tempi di attesa procurano, infatti, un maggiore consumo di risorse.

È quindi lecito, in conclusione, porsi la seguente domanda: “Qual è l'utilità di conoscere e contenere il costo del lavoro di singole operazioni quando il costo del personale è, prevalentemente, un costo fisso per l'ospedale ovvero indipendentemente dalle diverse attività svolte" (Bondonio et al,1995). Nel nostro caso l'adozione di ketorolac consente, (senza aumentare nessun tipo di costo) di liberare delle risorse di lavoro. L'uso del ketorolac può ridurre non solo il costo d'acquisto dell' analgesico, ma anche i tempi di somministrazione e di permanenza al Pronto Soccorso. È naturale chiedersi se questi risparmi siano reali o potenziali, e la risposta dipende dall'uso che si farà dei tempi di lavoro così liberati. Infatti, solo nel caso in cui l'ospedale sia in grado di farne buon uso, si potrà affermare che tale risparmio potenziale si sia trasformato in risparmio effettivo (Bondonio et al,1995). L'impiego di una terapia farmacologica in una realtà ospedaliera che consente di liberare risorse di personale dovrebbe determinare contestualmente un adeguamento dei processi organizzativi interni. Questo risulta particolarmente evidente se si considera che le risorse umane, se ben gestite e valorizzate, sono il contributo più prezioso di cui dispone un ospedale, in quanto sono in grado di caratterizzare l'offerta della intera struttura cui appartengono.

\section{BIBLIOGRAFIA}

1. Benedetti C. et al. Systemic analgesics. In Bonica II ed. The management of pain. $2^{\text {nd }}$. Philadelfia: Lee and Febiger, 1990;1640-1675.

2. Bondonio P., Eandi M., (1995), Tempi e costi di lavoro associati al trattamento farmacologico: analisi di alcune opportunità di risparmio, in Farmeconomia, Vol. 2 n³.

3. British Medical Association. Royal Pharmacutical Society of Great Britain. British national formulary. London: BMA, RPS, 1997, ( $\left.\mathrm{N}^{\circ} 52\right)$.

4. Brown CR. et al. Comparison of intravenous ketorolac tromethamine and morfine sulphate in the treatment of postoperative pain. Pharmacoterapy 1990; 1640-1675.

5. Cordal WH et al. Comparison of intravenous ketorolac, meperidine and both (balanced analgesia) for renal colic. Ann Emerg Med 1996; 28: 151-158.

6. De Nicola A, Varrassi G.Farmacoeconomia e terapia antalgica. vol. 78, n. 2, Int. Journal of Pain Therapy, novembre 1997.

7. De Nicola A, Farmacoeconomia. Atti XIX Congresso Nazionale AISD, Milano 1997. 
8. Drummond M.F., O’ Brien B., Stoddard G.L., Torrance G.W., (1997), Methods for the economic evaluation of health care programmes, Oxford University Press, second edition.

9. Eandi M et al. Farmacoeconomia e terapia antibiotica. ABE Edizioni Scientifiche, 1993.

10. Eisenberg J et al. Assessing the hiden cost of antibiotic therapy for hospitalized patients. Drug Information J 1988; 22:459-469.

11. Gillis JC. et al. Ketorolac. An appraisal of its pharmacodynamic pharmacokinetic properties and therapeutic use in pain management. Drugs 1997; 53 180-189.

12. Goodacre SW et al. A protocol to improve analgesia use in the accident and emergency department. $\mathrm{J}$ Accid Emerg Med 1997; 13:177-9.

13. Jefferson T., Demicheli V., Mugford M., (1998), La valutazione economica degli interventi sanitari, Il pensiero Scientifico Editore, Roma.

14. Rainer et al. Cost effectiveness analysis of intravenous ketorolac and morphine for treating pain after limb injury: double blind randomised controlled trial. BMJ 2000; 321 1247-1251.

15. Yealy DM. et al. Ketorolac in the treatment of acute pain. The peridulum swing. Ann Emerg Med 1992;21:985-986. 